\title{
HOLOGRAPH CONTRACTION BY OSCILLATORY FILTERED LEARNING FOR DYNAMIC SUB-PATTERN MATCHING
}

\author{
Javed I. Khan \\ Department of Math \& Computer Science \\ Kent State University \\ 233 MSB, Kent, OH 44242 \\ Javed@kent.edu
}

\begin{abstract}
The paper presents a scheme for reducing memory space of a holographic associative memory for content-based learning, searching and retrieval of sparse patterns. Multidimensional holographic associative memory developed on the properties of complex valued Riemann space is one of the most promising models of associative memory. It has demonstrated the unique ability to perform dynamically localizable sub-pattern matching, without requiring to learn each individual sub-patterns. The correlation space of the sparse patterns, is also sparse in information, but representationally dense. Therefore, holograph of sparse patterns (such as images) becomes extremely large. In this paper we describe a holographic memory model which can prune a holograph by several fold. The resulting holographic model also simultaneously increases the encoding, searching and decoding speed.
\end{abstract}

Keywords: partial-pattern matching, associative computing, holographic memory, target recognition, adaptive control.

\section{INTRODUCTION}

Since the advancement of synoptic theory of signal transmission by McCulloch and Pitts (1943), and Hebb (1949) a number of models of artificial associative memories have been developed to mimic the behavior of human brain by researchers such as Marr (1969), Anderson (1989), Willshaw (1971), Kanerva (1988) and many others [Carp89, HiAn85, Kane88, Will89]. These pioneering models were able to reproduce some of the intriguing behaviors of human brain.

Two of the most serious concern with most of these associative memories are their capacity and difficulty in storing arbitrary patterns. However, for image applications, the problem becomes more acute in terms of enormous physical memory requirement. Among the previous researchers, Kanerva [Kane88] has directly addressed the issue of physical space requirement. Also Khan and Yun have investigated feature based space contraction of memory using principal component analysis on patterns [KhYu94, Oja83].
A typical image pattern generally consists of a large number of pixels, and by nature individually they carry small amount of significant information. As a consequence, the correlation space of the image pattern becomes physically large but sparse in information. The objective of our research is to find ways to extract useful information from this sparseness of the correlation space and to contract the physical space of associative memory.

Our research is specifically aimed at a novel complex numeric representation based associative memory paradigm. In 1995 Khan [Khan95, Khan98] demonstrated a generalized computational model Multidimensional Holographic Associative Computing (MHAC), which demonstrated the unique ability of dynamic search localization. It has already been demonstrated that it can recollect the best match with respect to a dynamically defined sub-patterns, without requiring to learn subpatterns separately, even when the window of focus drops as low as $20 \%$. Research also demonstrated its superior speed and capacity performance [Khan95, Suth90]. Experiments have revealed speedup of factors 10 to 100 times compared to other paradigms.

The result is particularly important not only in associative computing paradigm, but also in general pattern matching applications because, multidimensional best-match partial pattern search degenerates to expensive exhaustive search even with known procedural algorithms [Khan95]. The model potentially opens up the power of associative computing to numerous complex pattern matching applications. This particular research focuses on the space contraction of the correlation substrate of MHAC. It presents the performance of a filtered reinforcement learning (FRL) algorithm that can offer the design tradeoff between space requirement and precision of reconstruction.

\section{HOLOGRAPHIC ASSICIATIVE COMPUTING}

\subsection{Representation}

MHAC represents information as a two tire quantity, the actual measurement and a meta-information "attention" 
(or focus). For example for an image the pixel value is the measurement. Each pixel may also take an associated second meta-quantity representing the "importance" of the pixel. Computationally, this bi-modal information is represented as a multidimensional complex number (MCN) spanned in a hyperspherical space. In this scheme an element of information is represented as:

$$
s_{k}=\left(\lambda_{k}, \alpha_{k}\right) \Rightarrow \lambda_{k} e^{\left[\sum_{j}^{d-1} \hat{i}_{j} \theta_{j, k}\right]}
$$

Here, $\alpha_{k}$ is the measurement and is mapped onto a set of phase elements $\theta_{j, k}$ in the range of $\pi \geq \theta \geq-\pi . \lambda_{k}$ is the meta-quantity focus. Following are the representations of a complete stimulus pattern and a response pattern:

$$
\begin{aligned}
& {\left[S^{\mu}\right]=\left[\lambda_{1}^{\mu} e^{\left[\sum_{j}^{d-1} \hat{i}_{j} \theta_{j, 1}^{\mu}\right]}, \lambda_{2}^{\mu} e^{\left[\sum_{j}^{d-1} \hat{i}_{j} \theta_{j, 2}^{\mu}\right]}, \ldots, \lambda_{n}^{\mu} e^{\left[\sum_{j}^{d-1} \hat{i}_{j} \theta_{j, n}^{\mu}\right]}\right]} \\
& {\left[R^{\mu}\right]=\left[\gamma_{1}^{\mu} e^{\left[\sum_{j}^{d-1} \hat{i}_{j} \phi_{j, 1}^{\mu}\right]}, \gamma_{2}^{\mu} e^{\left[\sum_{j}^{d-1} \hat{i}_{j} \phi_{j, 2}^{\mu}\right]}, \ldots, \gamma_{m}^{\mu} e^{\left[\sum_{j}^{d-1} \hat{i}_{j} \phi_{j, m}^{\mu}\right]}\right]}
\end{aligned}
$$

\subsection{Training and Retrieval}

Learning constitutes computation of individual complex associations, and superimposition of the associations on the holographic substrate. Following equation describes a reinforcement model of holographic learning:

$$
\left[X^{t+1}\right]=\left[X^{t}\right]+\eta \cdot[X][\bar{S}]
$$

The substrate $[\mathrm{X}]$ is stored as a MCN matrix. $\eta$ is the learning constant. The substrate acts as the memory. The retrieval process is similar to optical convolution. During recall, an excitatory stimulus pattern $\left[\mathrm{S}^{\mathrm{e}}\right]$ is obtained from the query pattern: In the event, that this new stimulus resembles closely to a priory encoded stimulus, then the corresponding response pattern is generated with high magnitude. The decoding operation is performed by computing the inner product of the excitatory stimulus and the correlation matrix $[\mathrm{X}]$ :

$\left[R^{e}\right]=\frac{1}{c}[X]\left[S^{e}\right]$, where $\mathrm{c}=\sum_{\mathrm{k}}^{\mathrm{n}} \lambda_{\mathrm{k}}$

The model treats the measurement component of information in a fundamentally different way than any NN. The elements of these vectors are complex numbers and measurement components are exponents. A complete theoretical and empirical analysis of the characteristics of this model can be found in [Khan95] and has appeared in [Khan98].

The underlying process can be explained through the recovery of a single response element, through (1a). Let the subscripts $\mathrm{i}$ and $\mathrm{j}$ refer to the element index and $\mathrm{t}$ refers to the association index. According to (1a) and (2), the jth element of the query response If $\left[S^{e}\right]$ is close to some priory encoded stimulus $\left[S^{(t=T)}\right]$, then the above equation can be rewritten as:

$r_{(i, e)}=\frac{1}{c} \sum_{i=1}^{n} r_{(j, t=T)} \bar{s}_{(i, T)} \cdot s_{(i, e)}+\frac{1}{c} \sum_{i=1}^{n}\left[\sum_{t \neq T}^{k} r_{(j,)} \bar{s}_{(i, t)}\right] \cdot s_{(i, e)}$

The phase of the first summation term here is exactly equally to the phase of $r_{(j, T)}$. Because, the product $\bar{S}_{(i, T)} \cdot S_{(i, e)}$ is always a scalar quantity. For symmetrically distributed associations, the second summation contributes as a random walk in the two dimensional vector space, The length of this path grows very slowly with the square root of the number of vectors. Thus, the resulting response phase closely resembles the phase of the correct response. If both the stimulus and response patterns are identical, then holograph acts as a content addressable auto associative memory. More extensive analysis of this holographic process can be found in [Suth92].

\subsection{Filtered Reinforcement Learning}

Now we will concentrate on the reduction of physical space required by the holograph $[\mathrm{X}]$. The basis for the reduction is given by the following equation, where the excitation of the holograph $[\mathrm{X}]$ is updated by the following equations:

$$
x_{i j}=x_{i j}+\left[r_{i}-\sum_{k=1} f\left(x_{i k}\right) \cdot s_{k}\right] \cdot \bar{s}_{j}
$$

where, $f(t)=t$ for $t<\beta$, else $=0$

In the oscillation mode, the non-linearity computation is deferred. Instead of computing it in every training cycle, rather a linear $f()=t$ is used and in every $\mathrm{p}^{\text {th }}$ training cycle, the nonlinear reinforcement learning is used. The idea behind the non-linearity is to suppress the positive reinforcement for the holographic cells which are timidly excited below the threshold $\beta$. In this model when a cell is weak, other cells in the same cooperation/competition cluster receives higher reinforcement, and grows. At the end of learning, a large number of Holograph cells excitation falls to very small excitation level. This feature can be exploited to compress the explicit representation of the holograph.

\subsection{Search Localization}

In general, this memory can accept focus mask both during encoding and retrieval. The masks are represented as the magnitude of the corresponding complex elements. The mask used during encoding is called the "assertion mask', and the mask used during recollection is called 


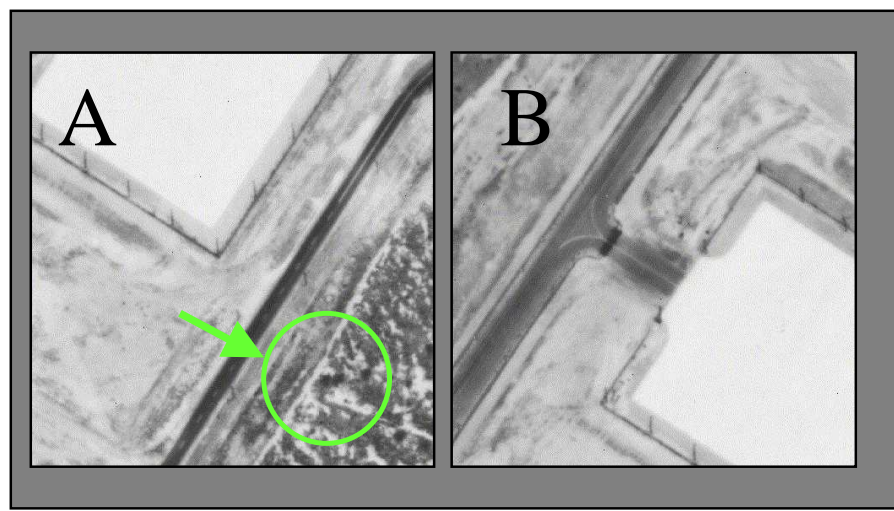

(a) Two archive photos A (left) and B (right)

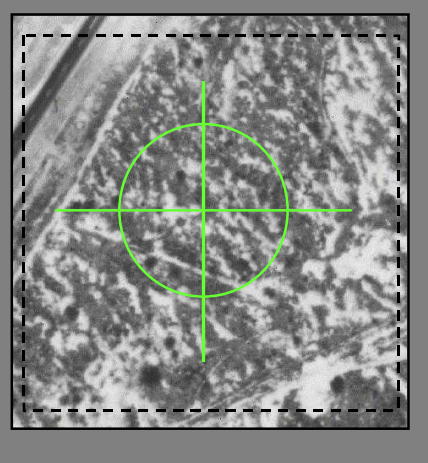

(b) Aircraft view

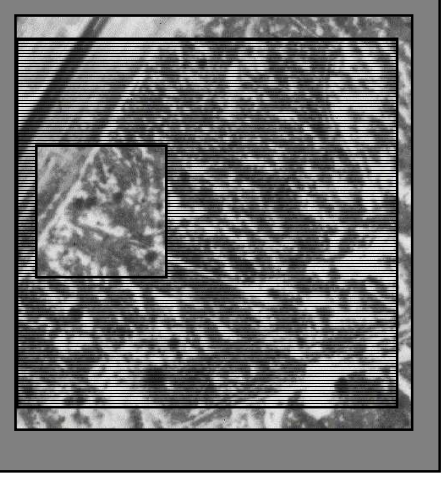

(c) Detection

Fig-1 Example of Holographic sub-pattern matching-based target recognition. (a) Examples of two archive photos. The target is at the intersection of an industrial complex and vegetated region. (b) The sample image taken by aircraft. ( c) Attentive masking on the target. This masked image finally matches image-A based on the focal zone. The localization ability of MHAC made it possible to retrieve correct match, despite the large differences between A and the sample.

"attention mask". Also this memory generates a complex output during recollection. The magnitude values of these elements are related to the pattern distance. A normalized average of the retrieved pattern strength can be interpreted as a 'confidence feedback' from the AAM for each retrieved pattern.

\section{EXPERIMENTS}

\subsection{Partial Pattern Matching}

In its simplest form, a large number of patterns are first "folded" into the correlation memory substrate of MHAC. It is called the holograph. During the encoding, each input pattern is first converted into an MCN stimulus pattern S. This pattern is associated with the MCN response pattern called Response Label Pattern (RLP).

During the recollection process, the memory receives a sample pattern and an attention mask. These two is used to obtain the MCN query pattern. Once the query pattern is constructed, the associative search mechanism of

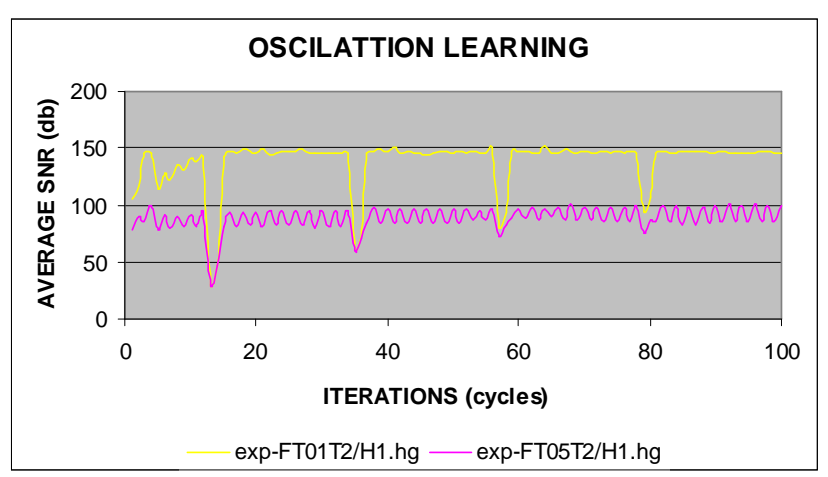

Fig-2 Oscillatory FRL convergence

MHAC performs associative recall. It returns an $\mathrm{MCN}$ pattern that corresponds to the RLP of the best match among the stored image. The recalled RLP pattern contains MCN elements with phases and magnitudes. A vector constructed out of the phase elements identifies the actual matching pattern. The magnitude vector corresponds to the MHAC's feedback confidence. High magnitude of the retrieved index pattern corresponds to potential match. Conversely, low magnitude corresponds to potential absence of the given object in the holograph.

Fig-1 illustrates the power of search localization and the method for target retrieval. As can be seen, the archived target images (A\&B), and the aircraft view (Fig-1(b)) with the target are quite dissimilar, because of the large difference in the surrounds and backgrounds. Unfortunately, a conventional AM depends on overall statistical image similarity and faces difficulty here.

As can be seen, MHAC has been able to dynamically (at query time) adopt to the focus mask to easily realize a localized search within the region of focus (Fig-1(c) ), and obtain the expected match. More interestingly, if needed a second target in the same aerial view can be locked in by just changing the region of focus during run time, without requiring the memory to re-learn the patterns. The intrinsically different complex representation provides MHAC this fundamental ability.

Although the above example illustrates the case of target recognition, the same novel search localization can be utilized in many other important application scenarios. Detection of small irregular patterns (medical diagnostics), detection of tiny targets, background varying target recognition, visual example based content-based image retrieval, robust adaptive control systems which needs to continue operating with small number of 


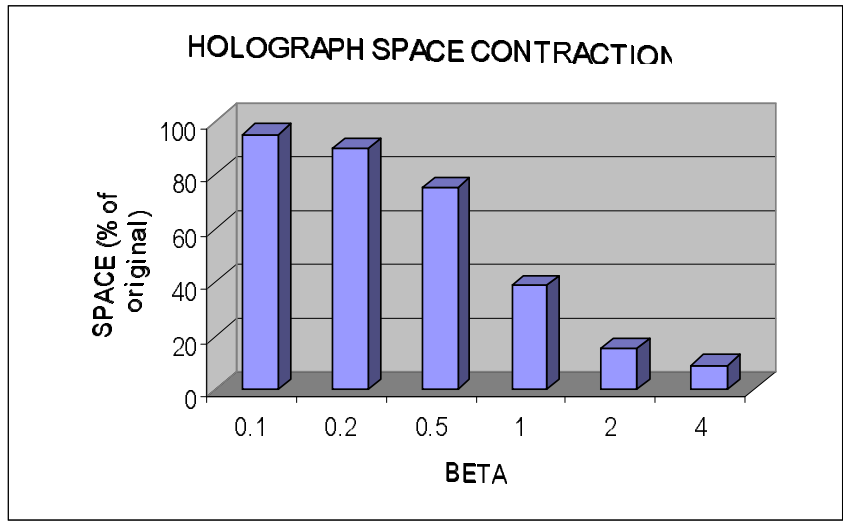

Fig-3 Space saving with high beta.

surviving sensors, in the face of post learning loss of sensors, are just few of the critical applications which can benefit from the model.

\subsection{Performance}

Below are the results of space reduction experiments are shown. In this scheme 100 patterns of sizes $100 \times 100$ were enfolded in a Holograph using the oscillatory FRL algorithm Fig-2 shows the convergence of the learning algorithm with training cycles for two beta values $(\beta=1$ and .5 respectively). The $\mathrm{Y}$ axis- plots the average accuracy of retrieval over all the learned patterns. The smaller the $\beta$, the better is the accuracy.

Fig-3 shows the average space reduction of the nonlinear reinforcement learning in the oscillation mode.

Fig-4 shows the space vs. retrieval accuracy performance trade-off. As evident, the holograph size can be pruned almost ten-fold (10\%), and still patterns can be retrieved with more than $30 \%$ accuracy.

\section{CONCLUSIONS}

The proposed work provides a formal mechanism for trade-off between size of space and quality of space for MHAC. As for other search problems, the reduction of search space also simultaneously reduces the search speed. The more is the sparseness of the pattern information, the less is the loss of quality in storage due to this contraction.

A further work will be to investigate how the holograph, which can be considered a highly compact archive of an enormous number of stored patterns, be coded for efficient transmission and recollection. Compared to feature based compaction [KhYu94], the actual number and distribution of excited cells are not pre-determinable in FRL algorithm. Variants of flexible techniques such as run length encoding can be used for compacting the holographic archive.

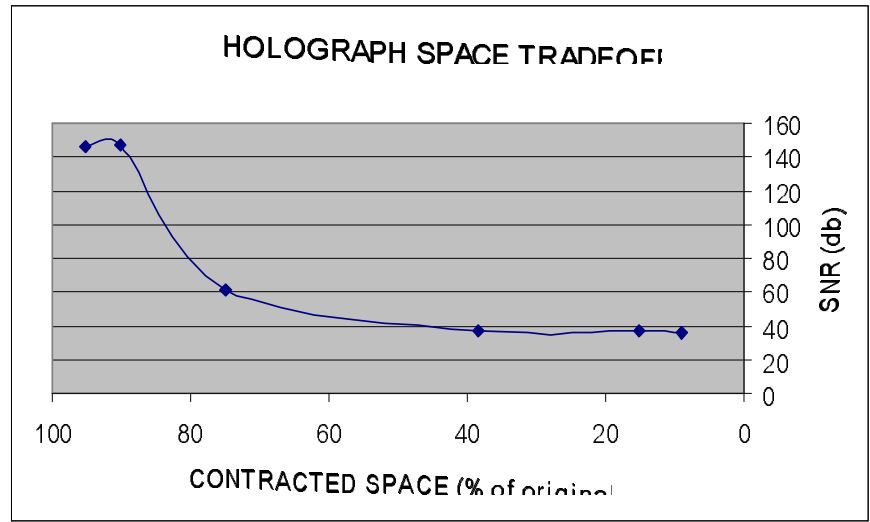

Fig-4 Space vs. RLP retrieval accuracy

One of the attractive characteristics of MHAC is the potential ability of real-time and fast retrieval in thousands of patterns. Its retrieval algorithms can be highly parallelized. Further research can be undertaken to investigate clever compaction schemes that will facilitate efficient block decomposition and fast computations in parallel recollection.

\section{BIBLIOGRAPHY}

[Carp89] Carpenter, G. A., "Neural Network Models for Pattern Recognition and Associative Memory", Neural Networks, v.2, 1989.

[Gabo69] Gabor, D., "Associative Holographic Memories", IBM J. of Research and Development, 1969, I3, p156-159

[HiAn85] Hinton, G.E., J. A. Anderson, Parallel Models of Associative Memory, Lawrance Erlbaum, NJ, 1985.

[Khan98] Khan Javed. I., Characteristics of Holographic Associative Memory in Retrieval with Localizable Attention, IEEE Transactions on Neural Networks, Vol-9, Issue-7, May '98, p.389-406.

[Khan95] Khan, J. I., "Attention Modulated Associative Computing and Content Associative Search in Images", Ph.D. Dissertation, Dept of Electrical Engineering, University of Hawaii, July, 1995.

[KhYu94] Khan J. I.,\& D. Yun, "Feature Based Contraction of Sparse Holographic Associative Memory", Proc. of World Congress on Neural Networks, WCNN'94, v.4, San Diego, June, 1994, pp26.

[Kane88] Kanerva, Pentti., Sparse Distributed Memory, MIT Press, Cambridge, 1988.

[Oja83] Oja, E., Subspace Methods of Pattern Recognition, John Wiley \& Sons, NY, 1983.

[Suth90] Sutherland, J. G., A Holographic Model of Memory, Learning and Expression, Journal of Neural Systems, v1, pp259-267, 1990.

[Will89] Willshaw, D., Holography, Associative memory and Inductive Generalization, Parallel Models of Associative Memory (updated edition), edited by G. E. Hinton, L. Erlbaum Associates, NJ, 1989. 
